\title{
The Efficacy of Using Synthetic Vision Terrain-Textured Images to Improve Pilot Situation Awareness
}

\author{
Michael D. Uenking \& Monica F. Hughes \\ NASA Langley Research Center
}

\begin{abstract}
The General Aviation Element of the Aviation Safety Program's Synthetic Vision Systems (SVS) Project is developing technology to eliminate low visibility induced General Aviation (GA) accidents. SVS displays present computer generated 3-dimensional imagery of the surrounding terrain on the Primary Flight Display (PFD) to greatly enhance pilot's situation awareness (SA), reducing or eliminating Controlled Flight into Terrain, as well as Low-Visibility Loss of Control accidents. SVSconducted research is facilitating development of display concepts that provide the pilot with an unobstructed view of the outside terrain, regardless of weather conditions and time of day. A critical component of SVS displays is the appropriate presentation of terrain to the pilot. An experimental study is being conducted at NASA Langley Research Center (LaRC) to explore and quantify the relationship between the realism of the terrain presentation and resulting enhancements of pilot SA and performance. Composed of complementary simulation and flight test efforts, Terrain Portrayal for Head-Down Displays (TP-HDD) experiments will help researchers evaluate critical terrain portrayal concepts. The experimental effort is to provide data to enable design trades that optimize SVS applications, as well as develop requirements and recommendations to facilitate the certification process.
\end{abstract}

In this part of the experiment a fixed based flight simulator was equipped with various types of Head Down flight displays, ranging from conventional round dials (typical of most GA aircraft) to glass cockpit style PFD's. The variations of the PFD included an assortment of texturing and Digital Elevation Model (DEM) resolution combinations. A test matrix of 10 terrain display configurations (in addition to the baseline displays) were evaluated by 27 pilots of various backgrounds and experience levels. Qualitative (questionnaires) and quantitative (pilot performance and physiological) data were collected during the experimental runs.
This paper focuses on the experimental set-up and final physiological results of the TP-HDD simulation experiment. The physiological measures of skin temperature, heart rate, and muscle response, show a decreased engagement (while using the synthetic vision displays as compared to the baseline conventional display) of the sympathetic and somatic nervous system responses which, in turn, indicates a reduced level of mental workload. This decreased level of workload is expected to enable improvement in the pilot's situation and terrain awareness.

\section{INTRODUCTION}

Synthetic Vision Systems (SVS) displays for General Aviation (GA) applications are designed to enhance pilot's situation awareness (SA), radically reducing the occurrence of Controlled Flight into Terrain (CFIT) accidents in addition to reducing or eliminating pilots' spatial disorientation (SD), thus preventing low-visibility induced Aircraft Upset (LVAU) events. LVAU events without recovery can lead to Low Visibility Loss of Control (LVLOC) accidents. Using imagery derived from terrain, obstacle, and airport databases, SVS displays provide the pilot with an unobstructed view of the outside terrain, regardless of weather conditions and time of day. In addition, through the integration of advanced symbology (i.e. highway in the sky, velocity vectors, etc.), navigation performance is drastically improved, with no effective increase in pilot workload.

The genesis of the Terrain Portrayal for Head Down Displays (TP-HDD) simulation experiment can be attributed to several factors. Actual requirements for terrain resolutions and texturing methods used to generate imagery on a PFD were previously undefined. In an attempt to utilize current technology avionics platforms with limited computational power, industry SVS displays have evolved with relatively low terrain portrayal fidelity that appear to be achieving substantial improvements in pilot performance over conventional displays (at the time this paper was written, references were not available to document GA industry SVS 
performance improvements). These new industry SVS displays utilize low to medium Digital Elevation Model (DEM) resolution databases (in the 30 arc-sec range) and simple terrain texturing concepts. In contrast, the RTCA (Special Committee 193 (SC-193) and EUROCAE Working Group 44 (WG-44)) have prescribed database resolution requirements intended to meet foreseeable aviation applications that are much higher than current industry SVS applications. The RTCA committee recommends 3 arc-sec DEM for en route operations, 1 arc-sec DEM for terminal operations, and .3 arc-sec DEM for surface operations [1]. As a result, an inconsistency exists between GA industry SVS DEM data usage and the RTCA recommendations. In addition, there is a pervasive belief among various elements within the aviation community that highresolution DEM data always provides significantly superior pilot SA and better pilot performance than lower-resolution DEM data. This belief is fueled, in part, by elaborate pictures of high-fidelity terrain portrayal SVS concepts. While higher fidelity terrain portrayals can be applied to SVS terrain displays, there is an inherent increase in SVS system complexity and cost. Previous SVS research has demonstrated that various concepts can work; however, proportional benefits of high-fidelity SVS imagery were unknown prior to this effort. In-depth research to help define the terrain portrayal requirements for SVS displays is needed. The combined TP-HDD simulation and flight efforts explores these issues in order to attempt to generate critical data that will provide a basis for recommendations appropriate for all SVS applications, with a focus on GA.

While previous studies have been conducted regarding the understanding of SA and SD, leading to some novel concepts, only relatively minor progress has been made towards measuring the effectiveness of these advanced flight display concepts [2-8]. As an initial investigation, the SVS-GA team conducted a study that focused on determining the associated benefits of SVS displays towards reducing LVLOC and CFIT accidents for GA pilots [2]. Results of that fixed-based simulator study demonstrated the effectiveness of generic SVS displays as compared to conventional GA flight decks in reducing pilot errors and thus improving pilot ability to control the aircraft during IMC. The current study builds upon results from reference 2 and in addition provides evaluation of multiple SVS display concepts.

\section{OBJECTIVES OF THE TP-HDD EXPERIMENT}

One of the primary challenges of SVS as a PFD is the presentation of appropriate information in a cost effective and computationally viable manner. The TPHDD experiments focus specifically on the aspect of portraying terrain effectively on a PFD. As previously mentioned, the TP-HDD study consisted of two distinct efforts. The first (current study) focused on flight simulation while the subsequent phase of the study was a flight experiment, employing the NASA LaRC Cessna 206 research aircraft.
The objectives of the TP-HDD simulation experiment were to establish the relationship between terrain depiction fidelity and pilots' terrain SA, pilots' performance (control and navigation), and prevention of LVLOC/LVAU incidents. Another objective of this study was to demonstrate that non-instrument rated pilots were able to fly approaches to an acceptable level of precision, with minimal training, using an SVS PFD with tunnel symbology. Flight-testing was employed to confirm and extend simulation results. The final overall goal of TP-HDD testing was to further establish the overall benefit of SVS for GA pilots.

Several measures were recorded in order to determine levels of workload and situation awareness during the TP-HDD experiment. In addition to performance data, NASA Task Load Index (TLX) ratings were collected, as well as Situation Awareness Rating Technique (SART) measures and Cooper-Harper ratings. Physiological data were recorded and analyzed as a supplement to these other types of measures, providing a means for interpreting the data in order to draw viable conclusions.

In addition, this experiment directly provided support for the Federal Aviation Administration (FAA) Capstone-2 certification issues. The Capstone-2 program is endeavoring to develop and supply new and emerging avionics technologies to the commercial aircraft fleet in Juneau, Alaska, to reduce the extremely high-rate of aircraft accidents in that area. One technology being employed for Capstone-2 is SVS displays.

\section{METHOD}

The experiment was conducted using a fixed-based simulator equipped with a strategic (navigation) and a tactical (primary) flight display. Various terrain portrayal concepts were developed from combinations of DEM resolutions and texturing concepts for the PFD. These displays were evaluated in the General Aviation WorkStation (GAWS) facility at NASA Langley Research Center. Twenty-seven subject pilots participated in the TP-HDD simulation experiment.

\section{DISPLAY CONCEPTS}

The display concepts evaluated can be grouped into three types. One type of display replicated conventional PFD's and was referred to as the blue-sky/brown ground (BSBG) concept. PFD's feature integrated information (i.e. airspeed, altitude, attitude) into one display (see Figure 1). 


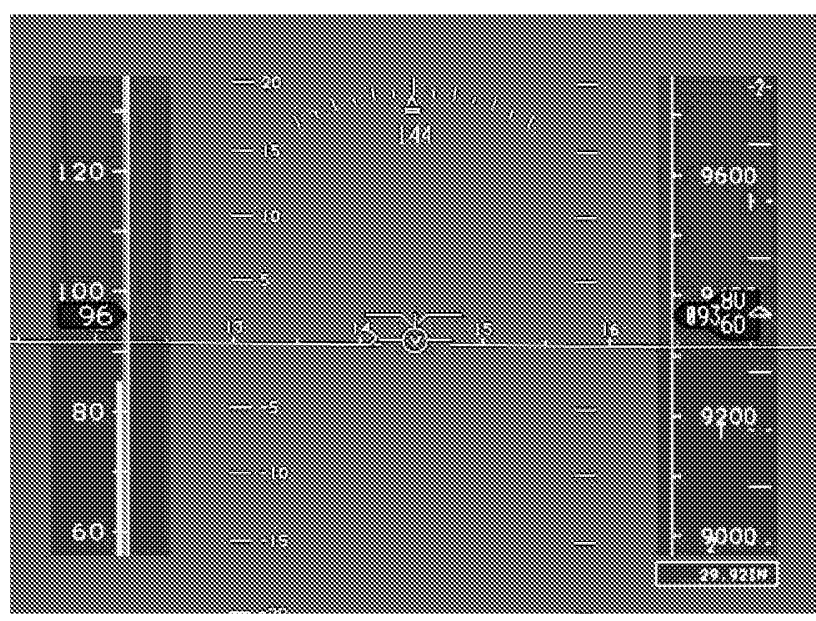

Figure 1. SVS-BSBG Baseline

Another type of display employed for this test was referred to as the baseline round dials (BRD) and is shown in figure 2 . The round dial concept replicates instrumentation currently found in the vast majority of GA aircraft.

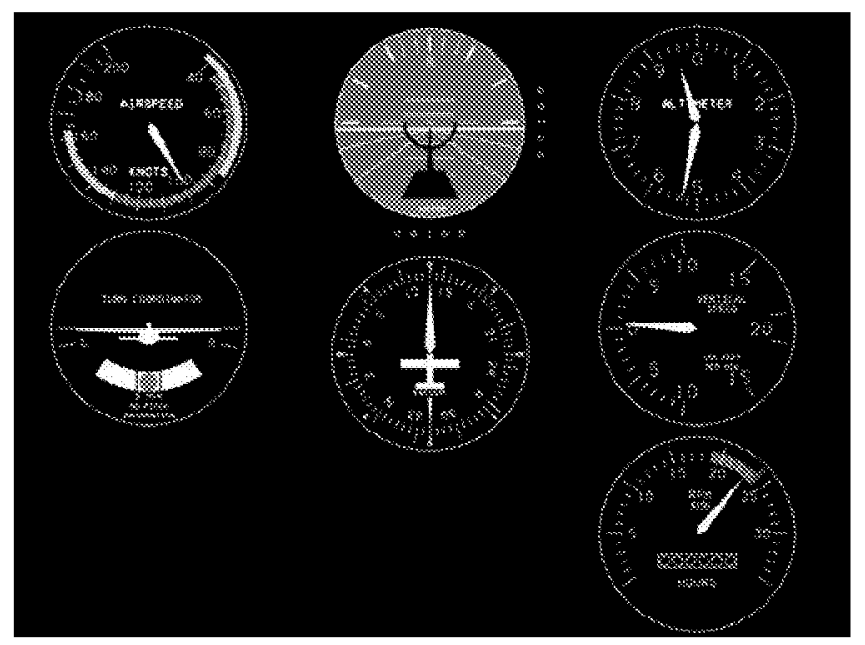

Figure 2. C172 BRD

The third type of display evaluated is referred to as the SVS display concept. SVS display concepts were identical to the conventional PFD, with the addition of the various SVS terrain portrayals in place of the bluesky/brown-ground. Figure 3 is an example of one of these SVS display concepts, with the Photo Realistic (PR) texture and DEM = 1 arc-sec.

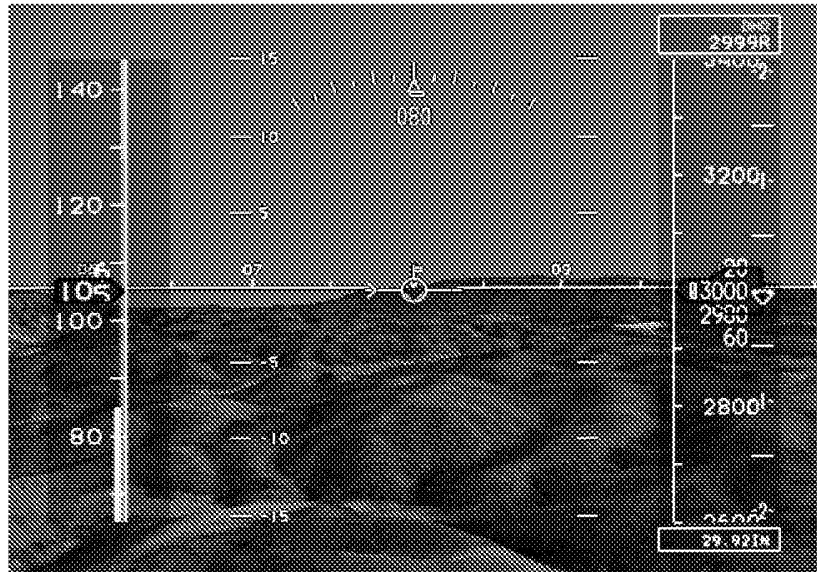

Figure 3. SVS Display Concept with Terrain

\section{TERRAIN DATABASES}

Encompassing the Roanoke Regional Airport (KROA), the simulated area of operations, termed the ROA Sector, was located in a relatively mountainous area in Virginia (VA), and was defined by the boundaries of $37^{\circ}$ $30^{\prime} \mathrm{N}, 79^{\circ} 40^{\prime} \mathrm{W}, 37^{\circ} 00^{\prime} \mathrm{N}$, and $80^{\circ} 40^{\prime} \mathrm{W}$. The SVS display concepts developed were combinations of various Digital Elevation Model resolutions (from the ROA Sector) and terrain texturing concepts.

\section{Digital Elevation Model Resolutions}

DEM resolution defines the distance between elevation data points (post-spacing) for a given a given database. Three specific DEM resolutions were chosen for the TPHDD experiment with the intent to cover a broad range of currently viable DEM options. The low resolution, 30 arc-sec $(900 \mathrm{~m} / 2953 \mathrm{ft}$ post-spacing) DEM was selected because it is currently being used in industry, and is a freely available database. The medium resolution, 3 arcsecond $(90 \mathrm{~m} / 295 \mathrm{ft}$ post-spacing) DEM and the high resolution, 1 arc-sec (30m/98ft post-spacing) DEM options were investigated in this experiment as a result of both of these resolutions being part of the set of DEMs prescribed by the RTCA and form an upper bound for current consideration.

It should be noted that the higher resolution databases are much larger in terms of overall data points for a given area of coverage, thus higher computational expenses are associated with manipulating these data. Since the databases for the lower resolutions are less populated than the higher resolutions, substantial terrain features might potentially be excluded. The possibility of losing entire peaks in the lower resolutions, as well as detailed terrain relief, exists. In Figure 4, a "rounded" effect becomes apparent between the $D E M=3$ and the $D E M=30$ (refer to the area indicated by the arrows in Figure 4). For this example, the elevation-based generic texturing concept is employed. 


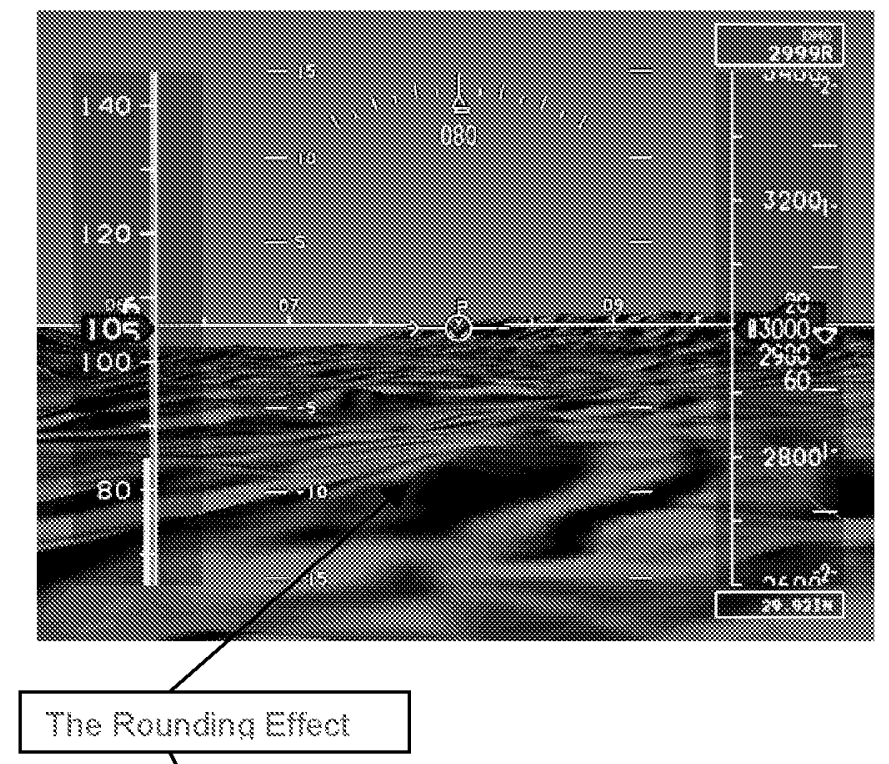

with darker colors, and higher terrain levels are assigned lighter colors. A certain shade of green was set to the field elevation, and the highest point was set to the highest terrain within $50 \mathrm{~nm}$ of ROA, approximately 4,000 $\mathrm{ft} \mathrm{MSL}$. The photo-realistic texturing was derived from 4 meter satellite imagery data that was draped over the various DEM databases. For the $C C$ and EBG terrain texturing display concepts, cultural features, such as roads, towers, and rivers, were presented on the display. Cultural and feature data were supplied directly through the photo-texture images on the PR displace concept.

\section{Fishnet (FN) Overlay Concept}

In addition to primary terrain texturing concepts, a fishnet grid overlay was employed. The theory of the fishnet grid involves placing grids of known sizes within the synthetic scene to facilitate pilot's depth perception. The potential benefits of the fishnet grid used in this study are cues for depth perception, distance, and angular rates. In addition, this fishnet grid provided a constant geometric shape in the synthetic terrain scene. However, the fishnet grid may introduce elements of confusion in terms of adding to display clutter, distracting the subject pilot, and being mistaken for the roads and rivers. These types of issues were investigated in this experiment. The spacing of the fishnet overlay was $500 \mathrm{ft}$ by $500 \mathrm{ft}$, regardless of the DEM resolution. The grid was dual-color (gray/white), to compensate for different coloring of features within the terrain databases (i.e., lighter colors of populated areas for the PR texture) and was constant for all texturing concepts.

\section{SYMBOLOGY}

\section{Primary Flight Display}

The SVS PFD featured several advanced symbology elements, including air-data, orientation, and guidance information. Air-data information was presented by use of integrated airspeed and altitude tapes with instantaneous digital values. An analog vertical speed indicator was integrated into the altitude tape. A roll pointer with a sideslip wedge and magnetic heading digital read-out portrayed heading, while a pitch ladder portrayed attitude orientation information. Additional symbology components displayed on the PFD were elements that characterized the velocity vector cluster. This cluster depicted current aircraft flight path angle and track angle, incorporating a non-quickened velocity vector with an acceleration-along-flight-path indicator (off the left finlet of the velocity vector marker) and sideslip flag (off the top finlet of the velocity vector marker). Guidance symbology was presented in the form of course deviation indicators showing glide slope and localizer deviation dots and a tunnel in the sky type of symbology (see Figure 5). The tunnel in the sky concept featured 400 feet wide by 320 feet tall, uniform green boxes depicting the current flight path for the approach scenario, providing lateral and vertical path guidance. Vertical and lateral path deviation indicators provided the 
pilot with additional information regarding proximity of the aircraft to the center of the tunnel.

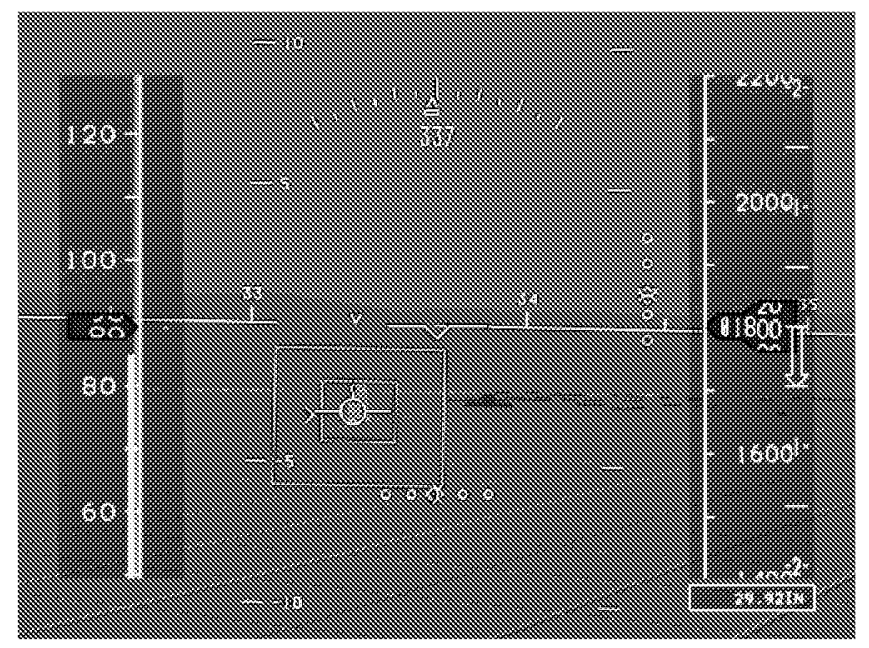

Figure 5. Example of the Constant Color with Fish Net, DEM $=\mathbf{3 0}$ arc-sec, with Tunnel

\section{$\underline{\text { Strategic Display }}$}

SVS perspective displays are designed to provide pilots with terrain $S A$ similar to and beyond the information provided by strategic terrain display concepts, such as the United Parcel Service Aviation Technologies (UPSAT) MX-20 multifunction display (MFD) (UPSAT $M X-20)$. The presence of the MX-20 in this experiment provided the capability to evaluate the integrated information supplied by both the PFD and the MFD, as well as estimate the relative value of each type of display. The MX-20 was used in the Terrain Awareness Mode only (see Figure 6), and was located in the radio stack. On the MX-20 MFD, terrain awareness, route information, waypoints, and towers were portrayed. All display concepts, including both baselines, were evaluated in the presence of the MX-20 MFD.

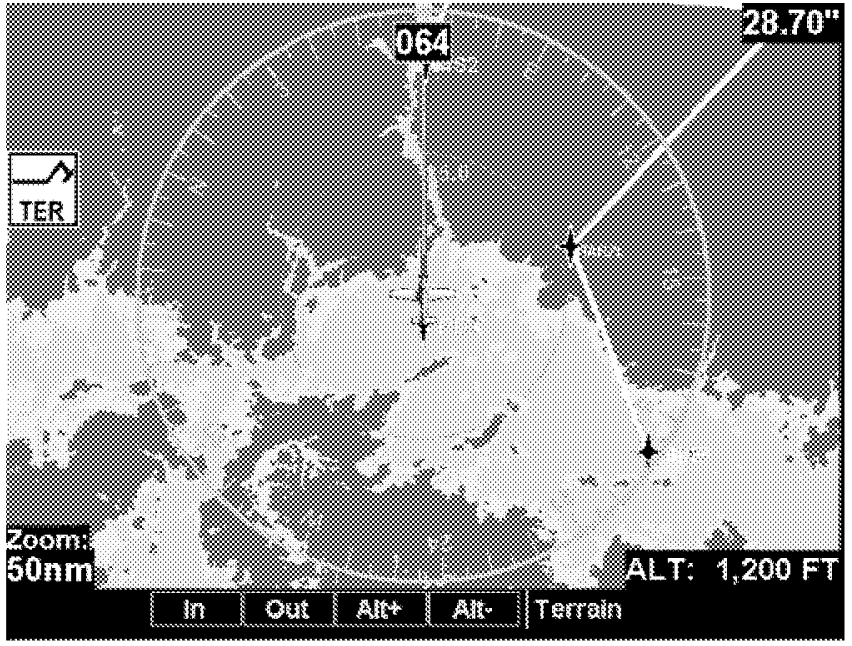

Figure 6. MX-20 MFD in Terrain Awareness Mode (image courtesy of UPSAT)

\section{TEST MATRIX}

Combinations of DEM and texturing concepts (including the $\mathrm{FN}$ option) produced 18 different potential terrain portrayal concepts to evaluate. A preliminary look at these 18 display concepts revealed that the $\mathrm{CC}$ texture without a FN provided little evident information on terrain contouring. As a result, the $\mathrm{CC}$ texture without the FN was eliminated as a feasible concept for terrain portrayal. For the remaining 15 concepts, a usability study was then conducted in order to down-select a smaller and more manageable set of candidate display combinations for more thorough investigation. While the formation of this set of candidate display concepts was based on subject pilot evaluations, the suggested collection of display concepts was broad enough to meet the objectives of the experiment. Subsequently, an adequate number of display-concepts (10) were retained for the core experiment (see Table 1).

\begin{tabular}{|c|c|c|c|c|c|c|}
\hline \multirow{5}{*}{ 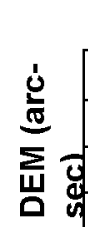 } & & \multicolumn{5}{|c|}{ Texturing Concepts } \\
\hline & & EBG & PR & CCFN & EBGFN & PRFN \\
\hline & 30 & & & $\sqrt{ }$ & $\sqrt{ }$ & $\sqrt{ }$ \\
\hline & 3 & & & & $\sqrt{ }$ & $\sqrt{ }$ \\
\hline & 1 & $\sqrt{ }$ & $\sqrt{ }$ & $\sqrt{ }$ & $\sqrt{ }$ & $\sqrt{ }$ \\
\hline
\end{tabular}

Table 1. TP-HDD Experiment Matrix (Legend: EBG=Elevation-Based Generic, PR=Photo Realistic, $\mathrm{CC}=$ Constant Color, $\mathrm{FN}=$ Fish Net)

These 10 SVS concepts were evaluated in addition to the specific baseline concept flight displays mentioned earlier. These two baseline flight displays, BSBG and BRD were split evenly between subject pilots with 
specific qualifications. All display concepts were randomized among the pilots for each maneuver.

One additional run was added to the above matrix for one specific texturing concept (Constant Color with Fish Net) and particular DEM (30 arc-sec), but without a tunnel during the approach maneuver. This trial was included in order to establish pilot performance and workload relationships regarding use of the tunnel in the sky guidance concept.

\section{EVALUATION PILOTS}

Twenty-seven evaluation pilots of various backgrounds and qualifications participated in the TP-HDD simulation and were grouped by certain characteristics. The first group of subjects consisted of fourteen low-time pilots with less than 400 hours and no instrument training beyond that required for the private pilot's license rating. Six instrument-rated pilots with various levels of experience, but less than 1000 hours, made up the second set of subjects. The last group of evaluation pilots consisted of four professional test pilots from NASA and the FAA and three Juneau (Alaska) area commercial operators. The Juneau pilots are potential future Capstone II users. In the physiological analysis, the last two pilot groupings were combined.

\section{SIMULATION EQUIPMENT}

The GAWS facility platform is based on a modified Precision Flight Control PC-based Aviation Training Device (PCATD) Model PI-142 instrument procedure trainer (see Figure 7). The model Pl-142 uses hardware typical of a general aviation aircraft with leftand right-pilot seats. Modifications to the hardware included the addition of a 6" SVS-PFD and a UPSAT MX-20 MFD.

Additionally, three different computers were employed to drive the system in GAWS. One computer, a Pentium-3 class PC, hosted the Initiative Computing (IC) Elite Electronic IFR Training Environment Version 6.2 software. This Elite software provided the aircraft dynamic responses to pilot control inputs, and control of the out-the-window weather, as well as data required to generate the research display imagery. Elite software can simulate various types of $\mathrm{GA}$ aircraft including a generic Cessna-172 model, which was used in this experiment. A Silicon Graphics Zx10 PC with a 3Dlab WildCat 4210 graphics card housed the SVS software employed to render the display presented on the SVSPFD. This SVS display software included the underlying databases with the various DEM resolutions and texturing concepts, combined with the SVS symbology. The third computer, another Silicon Graphics Zx10 PC with a 3Dlab WildCat 4210 graphics card, hosted the software that produced the out the window visual that was projected on the forward screen that provided the simulated out-the-window view.

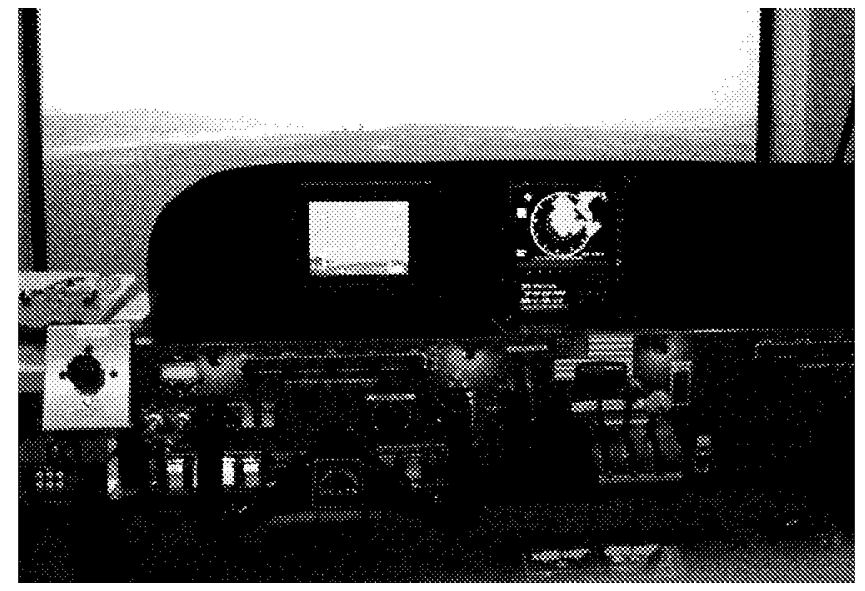

Figure 7. The General Aviation Workstation (GAWS) Facility Configured for TP-HDD

A pilot-selectable Display Field of View (FOV) control was developed for the experiment. Field of View is defined as the horizontal FOV of the image presented on the display. The four FOV choices for this experiment were $22^{\circ}, 30^{\circ}, 60^{\circ}$, and $90^{\circ}$. A minification factor (MF), which is the amount of angular compression to force an image to "fit" onto the display, is associated with each FOV. As the horizontal FOV increases, so does the MF. Higher MFs make terrain features appear further away than they are in reality. Two control options were available to the subject pilots, one located on the lefthand side of the instrument panel and one positioned on the right horn of the yoke. Previous research data indicate that a single fixed FOV would significantly limit SVS effectiveness. While flying each of the evaluation tasks, the subject pilots were encouraged to scroll through the FOV options during each phase of flight, evaluate the options, and provide comments during this experiment, as well as supply a resulting FOV strategy at the completion of the experiment.

\section{EVALUATION TASKS}

The evaluation tasks were developed to cover critical phases of flight. En route and approach phases of a flight were ranked as high priority for SVS applications by an earlier in-house study describing the SVS Concepts of Operation for GA [9]. To add some additional sensation of realism and more representative levels of workload, a low/moderate level of turbulence was simulated throughout the experiment. Due to anticipated higher levels of workload during the approach maneuver, physiological data was collected during this maneuver, only, and not during the en route scenarios. The complete test is described in reference 10. 


\section{$\underline{\text { Instrument Approach }}$}

The approach task consisted of a 6.5-minute flight simulation starting with a straight and level flight in VMC on a 30-degree localizer intercept course for the ILS (Instrument Landing System) 33 approach into ROA. The task started $12 \mathrm{~nm}$ south of ROA at 2,600 ft MSL, with a speed of $90 \mathrm{KIAS}$. The subject pilots were tasked to fly a heading of $300^{\circ}$ to join the localizer (approximately $10 \mathrm{~nm}$ from the threshold) and maintain $2,600 \mathrm{ft}$ until intercepting the glide slope at approximately $4.5 \mathrm{~nm}$, then continue flying the approach to $200 \mathrm{ft} \mathrm{AGL}$. In this case, out-the-window visibility was reduced from VMC to one statue mile within the first minute of the flight. In addition to moderate turbulence that decreased throughout the run, wind was simulated to be from $030^{\circ}$ at 15 knots, decreasing to 5 knots on final.

\section{$\underline{\text { Rare Event }}$}

The Rare Event task simulated a flight scenario with an incorrect altimeter setting. Effectively, the altitude tape indicated the incorrect (higher) altitude, which was different from the actual altitude portrayed by the terrain on the PFD. In addition, the altitude provided by the MX20 also included the $1,500 \mathrm{ft}$ error. This task was administered at the very end of the data collection for each evaluation pilot and was designed to look like the low altitude en route task. The starting point was at the same position as the low altitude en route task, but actual altitude was $1500 \mathrm{ft}$ lower. Consequently, the target level-off altitude for this maneuver was $500 \mathrm{ft}$ below ground level. Display concepts, excluding baseline concepts, were randomized among pilots, repeating one of the display concepts the evaluation pilot had already flown. The purpose of the rare event was to provide an experimental evaluation to establish whether or not the different SVS terrain concepts on the PFD provided terrain situation awareness to avoid CFIT accidents in a rare event.

\section{Operations}

The experiment was conducted within a 2.5 monthduration with no schedule interruptions. Each pilot participated in a day and a half of testing, consisting of approximately 35 trial runs, and 945 total trial runs for all pilots. Before the start of the experiment, each pilot received an extensive pilot briefing, as well as approximately one-hour of training time in the GAWS by a FAA certified flight instructor for instruments (CFII). The goal of these briefings and training was to familiarize each subject with the objectives of the experiment and educate the subjects on the salient features of the current SVS symbology set and the simulator functionality. The symbology set remained consistent across terrain databases for the approach maneuver, except for the tunnel-on and -off concepts. All high altitude En Route trials were completed during one block of 11 runs; all low altitude En Route trials were completed during the next block of 11 runs; and all approaches were completed in the third block of 12 runs. As previously stated, the rare event was typically the last run of the experiment for each subject pilot. Physiological data were collected only during the approach maneuver.

\section{PHYSIOLOGICAL DATA}

Physiological data is used as an added measure to provide a direct means of gauging a pilot's mental state while avoiding subjectivity inherent in questionnaires. It is also used due to the possibility that contamination of the other measured variables can occur [11].

Physiological state data such as heart rate (HRT), skin temperature (SKT), and muscle response (Electromyogram, EMG) were recorded to determine stress and workload during the instrument approach maneuvers. In addition to the collection during the approach maneuver, physiological data were documented for a limited number of subjects during the rare event as well, but only for evaluation (not statistical) purposes. The physiological data collection apparatus employed for this experiment was the MP100 ${ }^{\top \mathrm{M}}$ system developed by BIOPAC Systems Inc. (See Figure 8). Data were transmitted from the BIOPAC system to a Pentium-2 class PC for analysis.

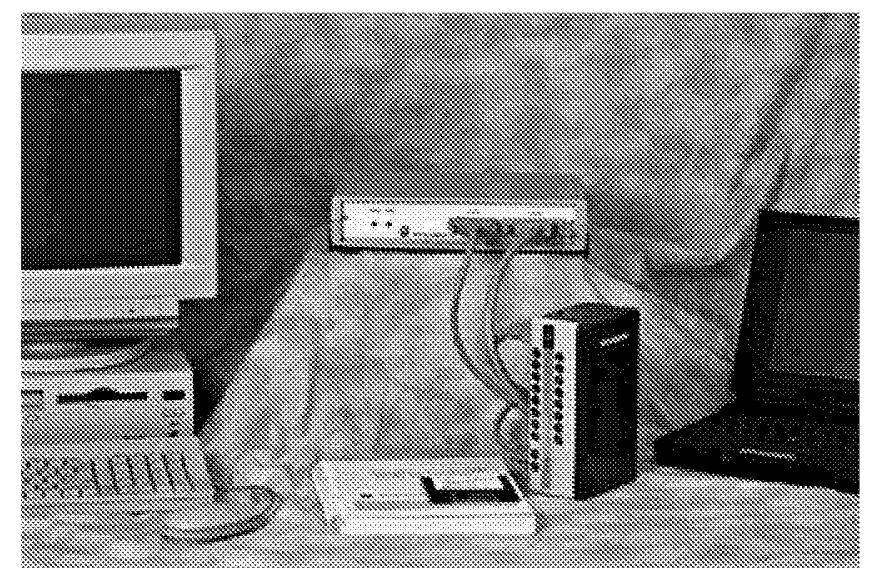

Figure 8. BIOPAC MP100"M Data Acquisition System

\section{Physiological Measures}

The first measure, HRT, was closely monitored to determine if the test subject's peak-to-peak heart rate changed due to the activities involved in the experiment. This heart rate change is the difference in time between each corresponding $\mathrm{R}$-wave peak. The $\mathrm{R}$-wave is the point at which the ventricles contract to force blood to the body's extremities from the heart muscle. This ventricular contraction produces the highest peak in the electrical signal that is shown in the data. Increases in 
HRT generally indicate increases in the level of stress and workload.

The second measure, SKT, was closely monitored to determine if the test subject's peripheral skin temperature changed relative to the experimental activities. In general, the peripheral skin temperature decreases in the situation of fight or flight to try to protect the major organs of the body. So, in the experimental setup, whenever a stressful situation occurs, a decrease in skin temperature will ensue.

The last measure, EMG, was closely monitored to determine if sudden muscle electrical activity occurs during the experiment. Whenever a particular muscle bundle is utilized, an electrical response occurs that is seen in the data as an electrical spike. For this experiment, increases in the time-integrated EMG signal can indicate increased levels of stress and workload.

\section{Electrode Placement}

Two sensors were used to measure HRT. The electrodes for heart rate were placed on each test subject as shown in Figure 9. The ground electrode was located behind the right anklebone on the instep side of the foot and is shown in Figure 10.

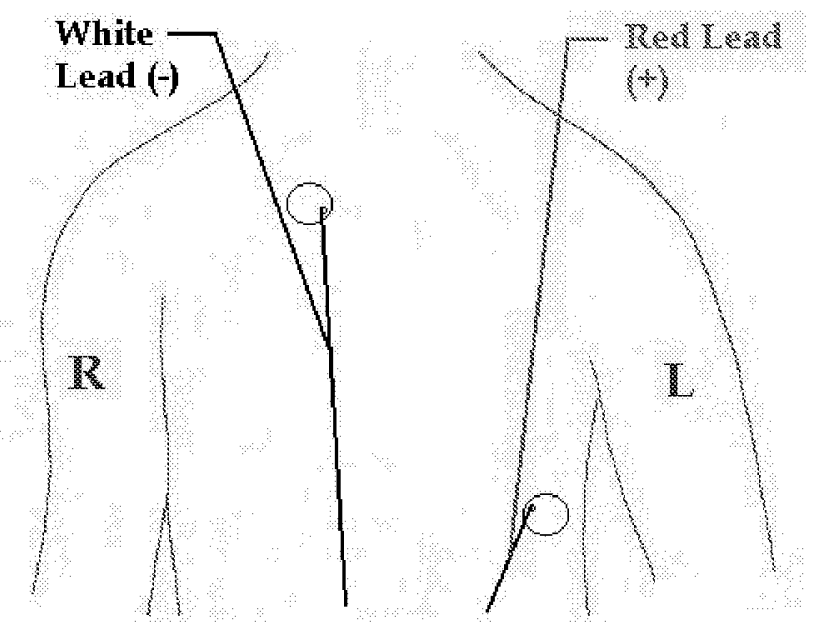

Figure 9. Heart Rate Electrode Placement

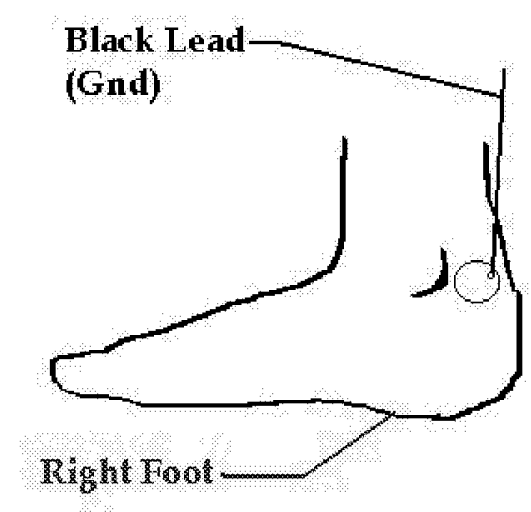

Figure 10. Ground Electrode Placement

The EMG procedures and sensors were the same as the ones used in the heart rate application. The two EMG sensors were placed on the left forearm as shown in Figure 11 below. The ground electrode was coupled with the same electrode used for the HRT.

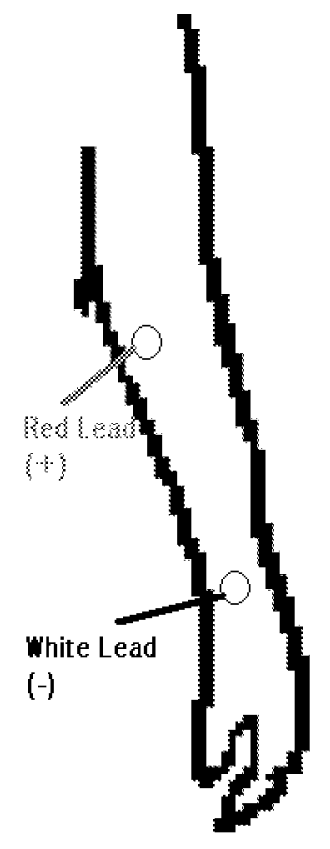

Figure 11. EMG Sensors Placement

For the skin temperature measurements, one small banjo-shaped temperature transducer was placed on the left ring finger and lightly attached via white surgical tape. 
All sensors were connected via the electrode cables into the BIOPAC amplifiers that were allocated for each individual physiological measure.

\section{RESULTS}

The physiological data were analyzed as part of this experiment to determine if the various display concepts produced measurable physiological differences that would indicate a change in their mental workload. Analyses were performed across all experience levels of subject pilots. However, only the skin temperature data from the low-time GA (VFR-rated) pilots showed significant results. These results are presented below.

\section{SKIN TEMPERATURE ANALYSES FOR THE APPROACH MANEUVER}

As seen in Figure 12, the mean delta skin temperature (DSKT) is lower for the synthetic vision displays vs. the BRD display. The delta values were calculated by measuring the difference between the resting physiological condition values vs. the IMC physiological condition values. The independent samples T-Test was employed to analyze the data using the SPSS ${ }^{\odot}$ software. The analysis was first employed to determine the significance of the largest difference in delta values as shown in Figure 12 (the comparison between the BRD vs. the Elevation-Based Generic, DEM = 1-arc-second (EBG1)). One can infer that, due to the statistical significance in the deltas between EBG1 and the BRD, workload using the SVS EBG1 display was decreased in comparison to the workload that these subjects experienced while using the BRD that they are more familiar with $(F(1,19)=-2.831, p=.011)$. Evaluating the next largest difference involved the comparison between the BRD vs. the Elevation-Based Generic with Fishnet, $\mathrm{DEM}=1$-arc-second (EBGFN1). The difference in these delta values was shown to be near significance $(F(1,19)=-1.919, p=.070)$. Further analyses of the comparisons between the remaining synthetic vision displays and the BRD showed no significance.

One thing to also note from Figure 12 is that there is a trend towards a decrease in DSKT from the BRD to the $B S B G$ and then subsequently to the EBG1. This would indicate that possibly not only did the terrain have an effect on the physiological state of the test subject, but also that the symbology that is included in the EBG1 and not in the BSBG could also be a factor as well.

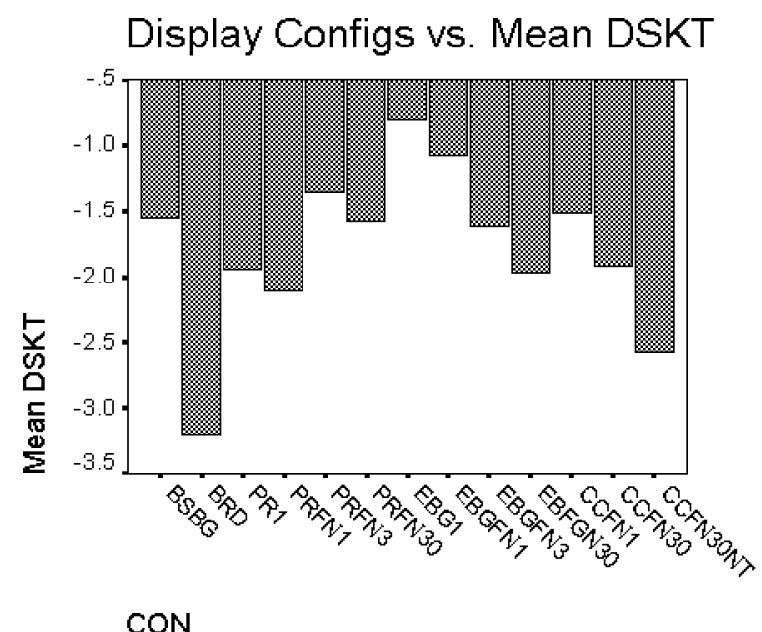

Figure 12. Graph of Mean Delta Skin Temperatures

Another skin temperature analysis was performed to determine the significance between the Constant Color with Fishnet, DEM $=30$ arc-second (CCFN30), with a tunnel concept, and the Constant Color with Fishnet, $\mathrm{DEM}=30$ arc-seconds, with No Tunnel (CCFN30NT). The tunnel versus no tunnel analysis for this particular SVS display (CCFN30) revealed no significance. However, if the CCFN30NT display concept was compared with the EBG1 display concept, significance was found $(F(1,26)=3.130, p=.004)$. This significance can be described as the combination of symbology and terrain effects. Likewise, comparing the CCFN30NT with the EBGFN1, it was very nearly significant $(F(1,26)=1.986, p=.058)$. These trends would indicate that the evaluation pilots experienced less stress and workload while flying using the EBG configurations which do utilize a tunnel vs. the CCFN30NT which does not.

\section{HEART RATE ANALYSES FOR THE APPROACH MANEUVER}

As illustrated in Figure 13, there was a decreased mean delta heart rate (DHRT) trend in the comparison between the BRD and the synthetic vision. However, the independent samples T-Test for significance failed to show any statistically significant findings. 


\section{Display Configs vs. Mean DHRT}

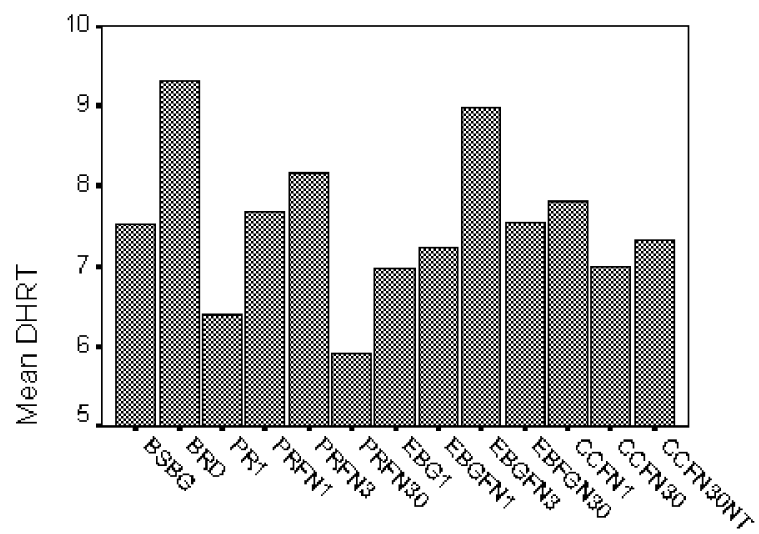

$\operatorname{CON}$

Figure 13. Graph of Mean Delta Heart Rate Values

\section{EMG ANALYSES FOR THE APPROACH MANEUVER}

The EMG data also failed to produce any significant findings other than that was found during the rare event maneuvers.

\section{PHYSIOLOGICAL RESULTS FOR THE RARE EVENT}

Throughout the duration of the experiment, during the rare event maneuver, a trend emerged indicating that the subject pilots were experiencing various levels of stress when coming into close proximity with terrain. As a result, the decision was made to collect physiological data on the last four evaluation pilots, during this rare event task. Data collection began during the descent, after the evaluation pilot completed the turn to the target heading (about 3.2 minutes into the run). For the example below, the pilot was approximately 1.8 miles from the terrain impact location at the end of the data. Figures 14-16 show the HRT, SKT, and EMG responses during the final leg of the rare event sequence.

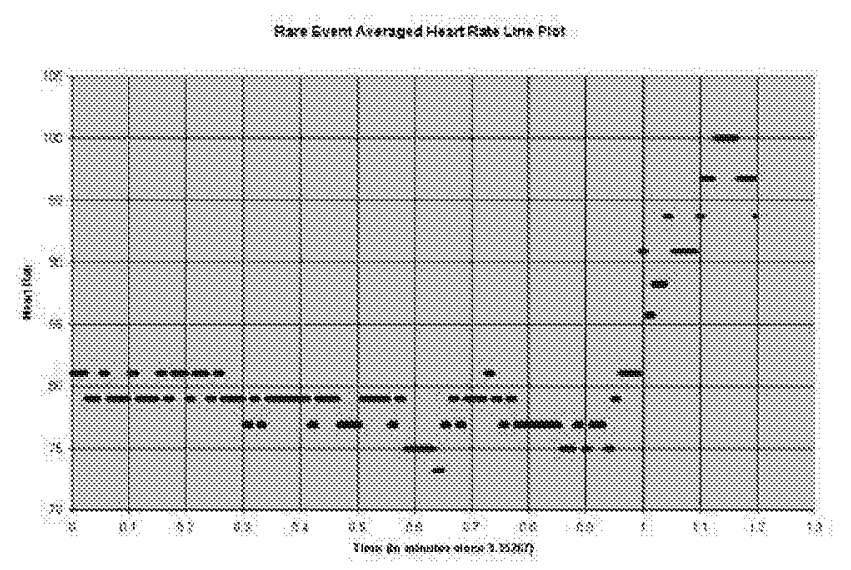

Figure 14. Graph of Average Heart Rate During Rare Event Sequence

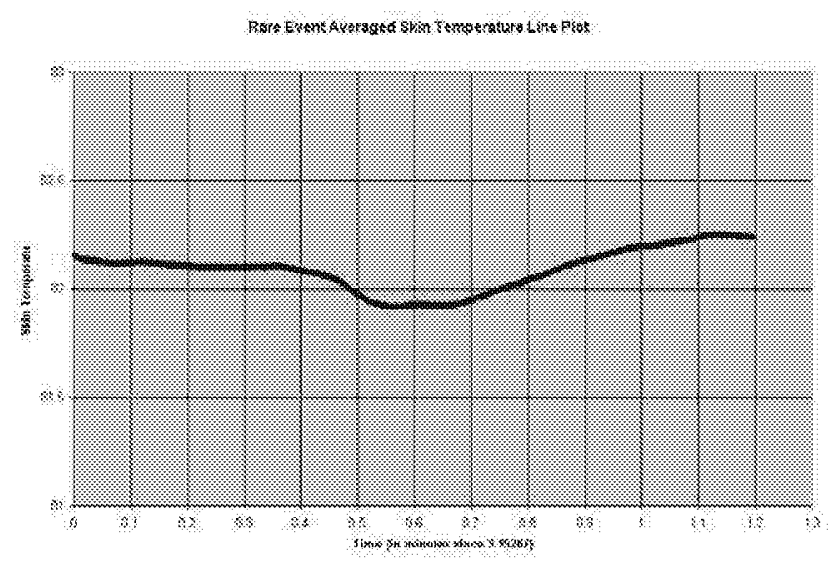

Figure 15. Graph of Average Skin Temperature During Rare Event Sequence

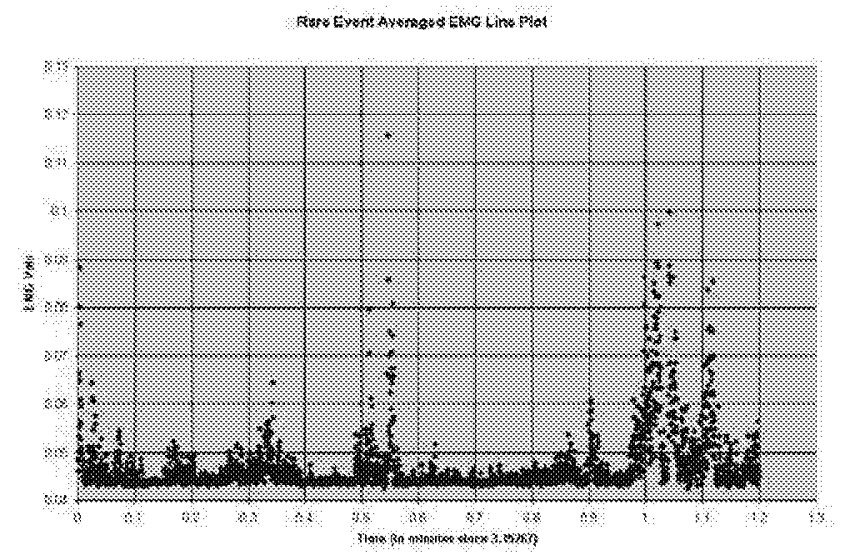

Figure 16. Graph of Average EMG Response During Rare Event Sequence 
During the rare event, the majority of the test subjects were highly engaged in utilizing the yoke to pull the aircraft up to avoid the terrain, and consequently, used more muscle response than during the more standard maneuvers. As the evaluation pilots flew closer to the terrain, each subject experienced an increase in heart rate, a decrease in skin temperature, and a more intense EMG response. The combination of these variables suggests that even though this was a simulation, conditions were realistic enough to induce some level of stress during this maneuver.

\section{CONCLUSION}

As seen in the physiological analyses for the VFR pilots, there was a measured decrease in mean Delta Skin Temperature (DSKT) and mean Delta Heart Rate (DHRT), indicating that the test subjects felt more comfortable with the synthetic vision displays vs. the standard gauges. As a result of the increased comfort level of the evaluation pilots, one can deduce that this indicates less workload. This decrease in workload would allow for an increase in cognitive resources that could be dedicated to study surroundings, thereby enabling the pilot to enhance situation awareness of the flying environment. With this enhanced Situation Awareness (SA), the pilot gains the important potential to avoid controlled flight into terrain or loss of control.

One other result that was noticed was that the test subjects preferred to have the displays in which the tunnel symbology was active. The CCFN30NT display was shown to be the third highest value in regards to DSKT only. The DSKT value comparison of the CCFN30NT with the EBG1 was shown to be very significant as well which helps to corroborate this finding.

The overall physiological responses of the evaluation pilots indicate that the subjects favored Synthetic Vision Systems (SVS) displays over Baseline Round Dials (BRD), in terms of terrain awareness, SA, and perceived pilot performance. Ongoing analyses of the pilot performance and the subjective questionnaires are currently being performed, with an expected result of corroborating these findings.

One of the thrusts of the experiment was to determine which display configurations would be more useful in enhancing situation awareness. Although significant results from one particular configuration (EBG1) vs. the BRD were apparent, comparing the individual SVS displays did not produce any statistical significant results. One possible reason for this was because all evaluation pilots were trained to fly the approach using the tunnel symbology, which leaves very little opportunity for the terrain to actually influence the subject's situation awareness.

Further examination of these types of display configurations with and without utilizing the tunnel symbology is recommended to provide an assessment of the effects each of these has on the pilot's workload and stress levels. This will isolate effects on the physiological data and provide a more realistic physiological response to the terrain depictions.

\section{ACKNOWLEDGMENTS}

The authors would like to thank Mr. Mark Rawls (NASA SHARP student, Crew Systems Branch) for his contribution in compiling the physiological data and the creation of the rare event graphs.

\section{REFERENCES}

1. Special Committee 193 (SC-193) and EUROCAE Working Group 44 (WG-44), 2002, User Requirements for Terrain and Obstacle Data, RTCA/DO-276, Washington, DC, pp. 1-94.

2. Glaab, L.J and M.A. Takallu, 2002, "Preliminary Effect of Synthetic Vision Systems Displays to Reduce Low-Visibility Loss of Control and Controlled Flight Into Terrain Accidents," SAE 2002-01-1550.

3. Abbott, T., M. Nataupsky, and G. Steinmetz, 1987, "Subjective, Physiological and Performance Measures of Eight Primary Flight Displays," Proceedings of OSU APS, 721-727.

4. Sachs, G., K. Dobler, and P. Hermle, 1998," Synthetic Vision Flight Tests for Curved Approach and Landing," TU Muenchen, IEEE 0-7803-5086-3.

5. Snow, M.P. and J.M. Reising, 1999, "Effect of Pathway-in-the-Sky and Synthetic Terrain Imagery on Situation Awareness in a Simulated Low-Level Ingress Scenario," Wright-Patterson AFB, ASC-991229.

6. Van Houten, Y.A., 1999, "Attentional Effects of Superimposing Flight Instrument and Tunnel-in-theSky Symbology on the World," National Lucht-en Ruimtevaartlaboratorium, NLR-TP-99141.

7. Williams, K.W., 2000, "Impact of Aviation HighwayIn-The-Sky Displays on Pilot Situation Awareness," FAA CAMI, DOT/FAAAM-00/31.

8. Vidulich, M.A. and E.R. Hughes, 1991, "Testing a subjective metric of situation awareness," Proceedings of the Human Factors \& Ergonomics Society 35th Annual Meeting, Human Factors \& Ergonomics Society, Santa Monica, CA.

9. Takallu, M. A., T.M. Doyle, F.G. McGee, M.F. Hughes, J.H. Koelling, and L.J. Glaab, 2001, "Concept of Operations for General Aviation Synthetic Vision Systems," NASA LaRC, Version 2.5 .

10. Hughes, M. F. and M. A. Takallu, 2002, "Terrain Portrayal for Head-Down Displays Experiment," IAATC, Anchorage, Alaska.

11. Wickens, C., S.E. Gordon, and Y. Liu, 1998, An Introduction to Human Factors Engineering, Addison Wesley Longman, Inc.: New York. 


\section{CONTACTS}

Michael D. Uenking, Human Factors Researcher, NASA Langley Research Center, MS 156A, Hampton, VA 23681-2199

(757) 864-6954

email: moduenkingolarcnasa nov

Monica F. Hughes, Deputy SVS-GA Lead, NASA Langley Research Center, MS 156A, Hampton, VA 23681-2199

(757) $864-3942$

email: M. Gughes dolarcnasa gov 